3. Electromotoric epidural vibration method.

4. Epidural exposure method.

Further, the concentrations of these enzymes were measured in C.S.F. from a series of patients with cerebrovascular disorders.

\title{
88. An Experimental Study on Cerebrospinal Fluid Circulation (Report III)
}

\author{
Akira SaIto \\ Second Surgical Division, Gifu Medical School
}

Concerning the circulation of cerebrospinal fluid (CSF), Dr. Suhara in our Department of Surgery has recently showed the CSF flowing from cisterna magna to spinal subarachnoid space slowly but constantly in dogs with spinal subarachnoid spaces blocked.

The present author has done similar experiments in dogs with basal cisternal blockage as produced by injecting Kaolin and Lycopodium suspension into the cisterna magna. The blockage extending from the cisterna magna to the basal subarachnoid space was established 2-3 weeks later in about $84 \%$ of dogs subjected to the injection. About $76 \%$ of dogs with the blockage developed an extensive or moderate hydrocephalus. In these hydrocephalic dogs a shunt operation was performed between lateral ventricle in the one side and cerebral subarachnoid space in the opposite side by the use of a fine polyethylene tube for the study of CSF circulation.

In eighteen dogs the basic CSF flow was found to be of av. $0.25 \mathrm{cc}$ per hour, showing an increase with the increase of ventricular pressure. When the ventricular pressure was raised artificially by the use of a T-tube interposed in the polyethylene tube, a parallel relationship was found to be present between the flow and the pressure under the condition of less than $250 \mathrm{~mm} \mathrm{H}_{2} \mathrm{O}$ of pressure at which point the flow seemed to reach a maximum level. Similar correlation between the pressure and the flow was noted even when only the subarachnoid spaces were loaded with certain pressure by physiologic saline solution. Therefore, it can be said that the increased flow subsequent to the elevated CSF pressure might be due to the accelerated absorption of water component in CSF.

The basic flow of CSF was decreased together with the decrease of pressure by intravenous administration of various hypertonic solutions, eventually showing the almost complete cessation at around $50 \mathrm{~mm} \mathrm{H} \mathrm{H}_{2} \mathrm{O}$ of pressure. How this change of CSF flow relate with production and absorption of CSF was not clear but in this series of experiments too there was a parallel relationship between the CSF 
pressure and the amount of flow.

Compression of the jugular vein so as to raise the intracranial venous pressure caused the back flow of CSF in combination with abrupt increase of the CSF pressure, being followed by return to normal flow after the release of compression. In this case an inverse proportion was found between the pressure and the flow.

From these experiments, it was concluded that the amount of CSF flow was related intimately to the CSF pressure, showing increase or decrease depending upon various factors contributory to the pressure, thereby a much complicated CSF dynamics being established as it is.

\title{
89. Ventriculo-Pleural Shunt for Hydrocephalus
}

\author{
Masashichi Kawano, Hikari Furuse, Jiro Moroki \& Hideaki Mori \\ Neurosurgical Service, Department of Surgery, Nagasaki University \\ School of Medicine, Nagasaki
}

No method of shunt for hydrocephalus is ideal and perfect, and even the ventriculo-auricular shunt, the latest entry, is not without fault. We have been trying ventriculo-pleural shunt for secondary hydrocephalus, using one-way silicon tubes with pressure control valve. So far only 4 cases were operated on; a 3 month-old hydrocephalic with a huge brain stem tetratoma, a 12-year-old boy with pinealoma, a 16-year-old girl with aqueduct ependymoma, and a 18-year-old young man with undetermined third ventricular tumor. No fatal complication was encountered. Obstruction was caused by slipped ventricular end of the tube and fibrin formation at the pleural end, and in each case correction was simple and easy requiring no general anesthesia.

From our experience we came to the conlusion as follows:

1. Ventriculo-pleural shunt is a safe and simple technic recommendable for primary as well as secondary hydrocephalus. 2. One-way silicon tube with pressure control valve of 50 to $70 \mathrm{mmH}_{2} \mathrm{O}$ is the best tube for this technic. 3. The route from (right) latral ventricle to pleural cavity via anterior 2 nd or third intercostal space is most suitable. 4. Young infant or patient with high-protein cerebrospinal fluid may develope dyspnea of various degree. 